\title{
Revealing Hidden Orbital Pseudospin Texture with Time-Reversal Dichroism in Photoelectron Angular Distributions
}

\author{
S. Beaulieu $\odot,{ }^{1, *}$ J. Schusser, ${ }^{2,3}$ S. Dong, ${ }^{1}$ M. Schüler, ${ }^{4}$ T. Pincelli, ${ }^{1}$ M. Dendzik $\odot,{ }^{1,5}$ J. Maklar $\odot,{ }^{1}$ A. Neef $\odot,{ }^{1}$ \\ H. Ebert, ${ }^{6}$ K. Hricovini, ${ }^{2,7}$ M. Wolf, ${ }^{1}$ J. Braun, ${ }^{6}$ L. Rettig, ${ }^{1}$ J. Minár, ${ }^{3, \dagger}$ and R. Ernstorfer ${ }^{1, \$}$ \\ ${ }^{1}$ Fritz-Haber-Institut der Max-Planck-Gesellschaft, Faradayweg 4-6, 14195 Berlin, Germany \\ ${ }^{2}$ Laboratoire de Physique des Matériaux et Surfaces, CY Cergy Paris Université, 95031 Cergy-Pontoise, France \\ ${ }^{3}$ New Technologies-Research Center, University of West Bohemia, 30614 Pilsen, Czech Republic \\ ${ }^{4}$ Stanford Institute for Materials and Energy Sciences (SIMES), SLAC National Accelerator Laboratory, \\ Menlo Park, California 94025, USA \\ ${ }^{5}$ Department of Applied Physics, KTH Royal Institute of Technology, Hannes Alfvéns väg 12, 11419 Stockholm, Sweden \\ ${ }^{6}$ Department Chemie, Ludwig-Maximilians-Universität München, Butenandtstrasse 11, 81377 München, Germany \\ ${ }^{7}$ LIDYL, CEA, CNRS, Université Paris-Saclay, CEA Saclay, F-91191 Gif-sur-Yvette Cedex, France
}

(Received 2 June 2020; accepted 13 October 2020; published 20 November 2020; corrected 14 December 2020)

\begin{abstract}
We performed angle-resolved photoemission spectroscopy (ARPES) of bulk $2 \mathrm{H}-\mathrm{WSe}$ for different $_{2}$ crystal orientations linked to each other by time-reversal symmetry. We introduce a new observable called time-reversal dichroism in photoelectron angular distributions (TRDAD), which quantifies the modulation of the photoemission intensity upon effective time-reversal operation. We demonstrate that the hidden orbital pseudospin texture leaves its imprint on TRDAD, due to multiple orbital interference effects in photoemission. Our experimental results are in quantitative agreement with both the tight-binding model and state-of-the-art fully relativistic calculations performed using the one-step model of photoemission. While spin-resolved ARPES probes the spin component of entangled spin-orbital texture in multiorbital systems, we unambiguously demonstrate that TRDAD reveals its orbital pseudospin texture counterpart.
\end{abstract}

DOI: 10.1103/PhysRevLett.125.216404

Locking between spin and valley degrees of freedom emerges in solids possessing a combined broken inversion symmetry and strong spin-orbit coupling, leading to peculiar valley-dependent spin texture in momentum space. This spin-valley locking leads to optical selection rules allowing for the generation of spin- and valley-polarized excited carriers [1-3], which can be used for all-optical selective spin injection [4,5]. In multiorbital systems, additional locking between crystal momentum and orbital degree of freedom emerges as a consequence of band hybridization, leading to complex entangled spin-orbital textures, as predicted in some topological insulators [6,7], two-dimensional electron gases [8], and transition metal dichalcogenides (TMDCs). The resulting momentumspace orbital texture can lead to the orbital Hall effect [9], the orbital Rashba effect [10], and the emergence of orbital Hall insulating phases [11]. Orbitronics [12], i.e., encoding (quantum) information in the orbital degree of

Published by the American Physical Society under the terms of the Creative Commons Attribution 4.0 International license. Further distribution of this work must maintain attribution to the author(s) and the published article's title, journal citation, and DOI. Open access publication funded by the Max Planck Society. freedom, can be seen as a newly emerging field, in analogy to spin- and valleytronics $[13,14]$.

TMDC monolayers are emblematic materials with entangled spin, orbital, and valley degrees of freedom. In a minimal electronic structure model of TMDC monolayers [15], the valence band at the Brillouin zone boundary $\left(K / K^{\prime}\right.$ points $)$ can be described by $\left|\psi_{\mathbf{k}}^{K / K^{\prime}}\right\rangle \approx\left\{\left[C_{0}(\mathbf{k})\left|d_{z^{2}}\right\rangle+C_{ \pm 2}(\mathbf{k})\left|d_{ \pm 2}\right\rangle\right] \otimes|\uparrow / \downarrow\rangle\right\}_{\mathrm{VB} 1}+$ $\left\{\left[C_{0}(\mathbf{k})\left|d_{z^{2}}\right\rangle+C_{ \pm 2}(\mathbf{k})\left|d_{ \pm 2}\right\rangle\right] \otimes|\downarrow / \uparrow\rangle\right\}_{\mathrm{VB} 2}$, where the label VB1 and VB2 represented the two first spin-orbit split valence bands and where $\left|d_{ \pm 2}\right\rangle=\left[\left|d_{x^{2}-y^{2}}\right\rangle \pm\right.$ $\left.i\left|d_{x y}\right\rangle\right] / \sqrt{2}$. While the spin texture is determined by the momentum-dependent spin state, the orbital texture is set by the momentum-dependent orbital pseudospin, defined as $\sigma_{i}^{K / K^{\prime}}(\mathbf{k})=\left\langle\psi_{\mathbf{k}}^{K / K^{\prime}}\left|\hat{\sigma}_{i}\right| \psi_{\mathbf{k}}^{K / K^{\prime}}\right\rangle$, where $\hat{\sigma}_{i}(i=x, y, z)$ is the Pauli operator [16] and where $x$ and $y$ are in-plane and $z$ out-of-plane coordinates (normal to the surface).

$K$ and $K^{\prime}$ valleys are related to each other via the timereversal operator, i.e., $\hat{T}\left|\psi_{\mathbf{k}}^{K}\right\rangle=\left|\psi_{\mathbf{k}}^{K^{\prime}}\right\rangle$. In TMDC monolayers, swapping valley indexes (time reversal), thus, reverses both the spin and orbital textures. In bulk TMDC of $2 \mathrm{H}$ polytype, the adjacent layers are rotated by $180^{\circ}$ with respect to each other, leading to opposite and alternating local spin polarization and orbital texture between neighboring layers. This peculiar layered structure naturally introduces the concept of "hidden" spin and 
orbital texture, which exists within each layer but vanishes in the bulk, i.e., when the inversion symmetry of the crystal is restored [17]. Probing such hidden physical properties is experimentally challenging. While spin- and angle-resolved photoemission spectroscopy is now a wellestablished surface-sensitive technique to investigate hidden spin texture [18-20], an experimental technique allowing one to selectively and directly probe hidden orbital texture has not been established.

In this Letter, we perform extreme ultraviolet (XUV) angle-resolved photoemission spectroscopy (ARPES) of bulk $2 \mathrm{H}-\mathrm{WSe}_{2}$ for crystal orientations rotated by $\alpha=60^{\circ}$ with respect to each others $\left(\mathcal{R}_{60^{\circ}}\right)$ effectively acting as the time-reversal operator $(\hat{T}): \mathcal{R}_{60^{\circ}}\left|\psi_{\mathbf{k}}^{K}\right\rangle \equiv \hat{T}\left|\psi_{\mathbf{k}}^{K}\right\rangle=\left|\psi_{\mathbf{k}}^{K^{\prime}}\right\rangle$. We introduce a novel observable, time-reversal dichroism in photoelectron angular distributions (TRDAD), which probes the modulation of the photoemission intensity upon time reversal and which is shown to be sensitive to the orbital pseudospin texture, due to multiple orbital interference effects in photoemission. We show that TRDAD is free of any spurious contribution from experimental geometry, which typically complicates the interpretation of the (linear or circular) dichroism in ARPES. Our experimental results are in quantitative agreement with state-of-the-art fully relativistic Korringa-Kohn-Rostoker (KKR) ab initio calculations performed using the one-step model of photoemission [21,22] and with a tight-binding model, which allows us to investigate the microscopic origins of TRDAD. While we introduce this novel observable using the emblematic bulk $2 \mathrm{H}-\mathrm{WSe}_{2}$ crystal, our conclusions are fully general and TRDAD can be used to probe (hidden) orbital texture in any multiorbital systems.

The experimental apparatus features a tabletop femtosecond (fs) XUV (21.7 eV, $110 \mathrm{meV}$ FWHM bandwidth) beam line [23] coupled to a time-of-flight momentum microscope spectrometer (METIS 1000, SPECS GmbH); see Fig. 1(a). This detector allows for simultaneous detection of the full first Brillouin zone, over an extended binding energy range, without the need to rearrange the sample geometry [24]. More details about the experimental setup can be found elsewhere $[23,25,26]$ and in Supplemental Material [27]. As shown in Figs. 1(b)-1(e), we recorded the 3D photoemission intensity for two different crystal orientations, rotated by $60^{\circ}$ with respect to each other. Looking at the experimentally measured constant energy contours (CECs) for energy $E-E_{\mathrm{VBM}}=$ $-0.25 \mathrm{eV}$, one can see that the photoemission intensity is strongly anisotropic around each $K / K^{\prime}$ valley, describing "croissant-shaped" patterns. This has been recently explained as originating from interference between photoelectrons emitted from the transition metal $d$-type orbitals [28]. The azimuthal variation of the photoemission intensity around $K / K^{\prime}$ points, i.e., the orientation of the croissant, changes upon rotation of the crystal by $60^{\circ}$. A modification of the momentum-resolved photoemission

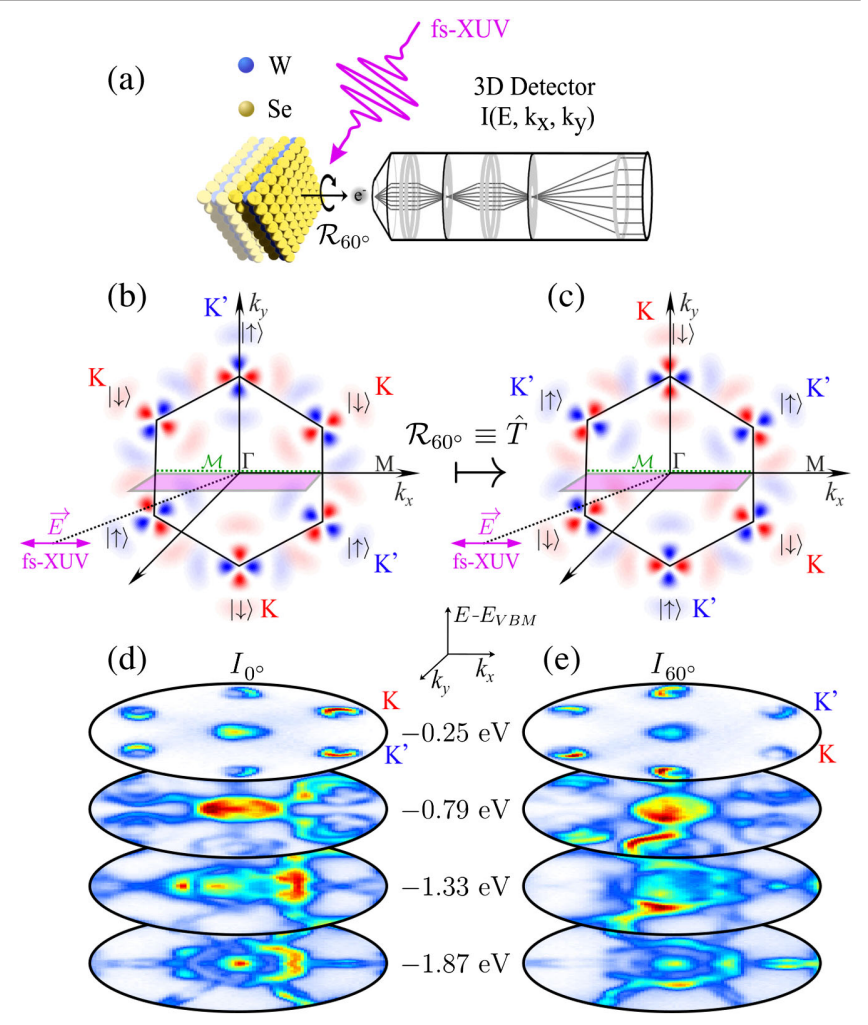

FIG. 1. Modulation of the photoemission intensity upon time reversal $(\hat{T})$. (a) Scheme of the experimental setup: A $p$-polarized femtosecond XUV $(21.7 \mathrm{eV})$ pulse is focused onto a bulk $2 \mathrm{H}$ $\mathrm{WSe}_{2}$ crystal at an angle of incidence of $65^{\circ}$ with respect to the surface normal, ejecting photoelectrons which are detected by a time-of-flight momentum microscope. (b),(c) Scheme of the experimental geometry and spin-orbital texture: The scattering plane (light purple plane) coincides with the crystal mirror plane $(\mathcal{M}$, green dashed line), which is along the $\Gamma-M$ high-symmetry direction. The spin-orbital texture is schematically represented by the orbitals and the up or down spin state at each $K$ or $K^{\prime}$ valley. A $60^{\circ}$ azimuthal rotation of the crystal $\left(\mathcal{R}_{60^{\circ}}\right)$ yields the transformation of $K$ to $K^{\prime}$ valley (and vice versa) and is analogous to the action of the time-reversal operator; i.e., $\mathcal{R}_{60^{\circ}}\left|\psi_{\mathbf{k}}^{K}\right\rangle \equiv$ $\hat{T}\left|\psi_{\mathbf{k}}^{K}\right\rangle=\left|\psi_{\mathbf{k}}^{K^{\prime}}\right\rangle$. (d),(e) Constant energy contours for different energies, $E-E_{\mathrm{VBM}}$, measured for the two different crystal orientations described above $\left(I_{0^{\circ}}\right.$ and $\left.I_{60^{\circ}}\right)$. The radius of each constant energy contour corresponds to $1.6 \AA^{-1}$.

intensity upon time reversal can also be seen for larger binding energies in Figs. 1(d) and 1(e).

Dichroisms in the angular distribution [both linear (LDAD) and circular (CDAD)] are powerful quantities relying on the modulation of the photoemission transition dipole matrix element upon the change of the ionizing radiation polarization state. $\mathrm{CDAD}$ has been used to probe electronic chirality in graphene [29], helical spin texture in a topological insulator [30], and Berry curvature in TMDCs [31,32], for example. LDAD is typically assumed to encode the nonrelativistic symmetry of the ground state wave function [33-35], which can contain information about the orbital texture [36,37]. However, dichroism can also 
have an extrinsic origin; i.e., it can also emerge from experimental geometry-induced symmetry breaking. Disentangling the intrinsic and extrinsic contribution to the dichroic signal is very challenging but of fundamental importance to extract meaningful physical insight from it.

Using our multidimensional detection scheme with the $p$-polarized femtosecond XUV pulses incident in the $k_{x}-k_{z}$ plane (and along $\Gamma-M / M^{\prime}$ ), the normalized intensity differences between the forward $\left[I_{\alpha}\left(k_{x}, k_{y}, E_{B}\right)\right]$ and backward $\left[I_{\alpha}\left(-k_{x}, k_{y}, E_{B}\right)\right]$ hemispheres, i.e., the linear dichroism asymmetry in the photoelectron angular distribution $\left[A_{\mathrm{LDAD}}^{\alpha}\left(k_{x}, k_{y}, E_{B}\right)\right]$, can be extracted [see Eq. (1)], without the need to rearrange the sample geometry or the light-polarization state $[38,39]$ :

$$
A_{\mathrm{LDAD}}^{\alpha}=\frac{I_{\alpha}\left(k_{x}, k_{y}, E_{B}\right)-I_{\alpha}\left(-k_{x}, k_{y}, E_{B}\right)}{I_{\alpha}\left(k_{x}, k_{y}, E_{B}\right)+I_{\alpha}\left(-k_{x}, k_{y}, E_{B}\right)} .
$$

Looking at $A_{\mathrm{LDAD}}^{0^{\circ}}$ and $A_{\mathrm{LDAD}}^{60^{\circ}}$ [Figs. 2(d) and 2(e)], one can notice that some features of the dichroism are invariant upon time reversal, while others show antisymmetric behavior (sign flip). This can be understood by the fact that the contribution to the dichroism originating from experimental geometry remains unchanged upon $60^{\circ}$ rotation of the crystal. Moreover, depending on the energymomentum region of the electronic structure sampled in each experimental data voxel, the associated ground state wave function might be invariant upon time reversal. For example, the dichroism emerging from the branches pointing along the $\Gamma-M / M^{\prime}$ high-symmetry direction, which is of multiorbital character $\left(\left|p_{x}\right\rangle \pm i\left|p_{y}\right\rangle\right)$, switches sign upon crystal rotation. On the other hand, the dichroism emerging from the hexagonal-shaped band surrounding the $\Gamma$ point, which is mostly of single orbital character $\left(\left|p_{z}\right\rangle\right)$, does not.

The new observable that we introduce, called timereversal dichroism in the photoelectron angular distributions (TRDAD), allows us to isolate the antisymmetric part of the $A_{\mathrm{LDAD}}$ dichroism upon time reversal, in order to remove any spurious contributions from experimental geometry. TRDAD is defined as

$$
\mathrm{TRDAD}=\frac{A_{\mathrm{LDAD}}^{\alpha}-A_{\mathrm{LDAD}}^{\alpha^{\prime}}}{2}
$$

where crystal rotation by an angle $\alpha-\alpha^{\prime}\left(\mathcal{R}_{\alpha-\alpha^{\prime}}\right)$ is equivalent to time reversal, i.e., $\mathcal{R}_{\alpha-\alpha^{\prime}} \equiv \hat{T}$. The resulting TRDAD is shown in Fig. 2(f). The branches pointing along $\Gamma-M$ have opposite $A_{\mathrm{LDAD}}$ with respect to adjacent valleys and dominates the signal, while the signature of the hexagonal-shaped band surrounding the $\Gamma$ point has disappeared. Indeed, a nonvanishing TRDAD signal implies that the state-resolved dichroism changes sign upon time-reversal operation, which we interpret as a switch of the orbital texture.
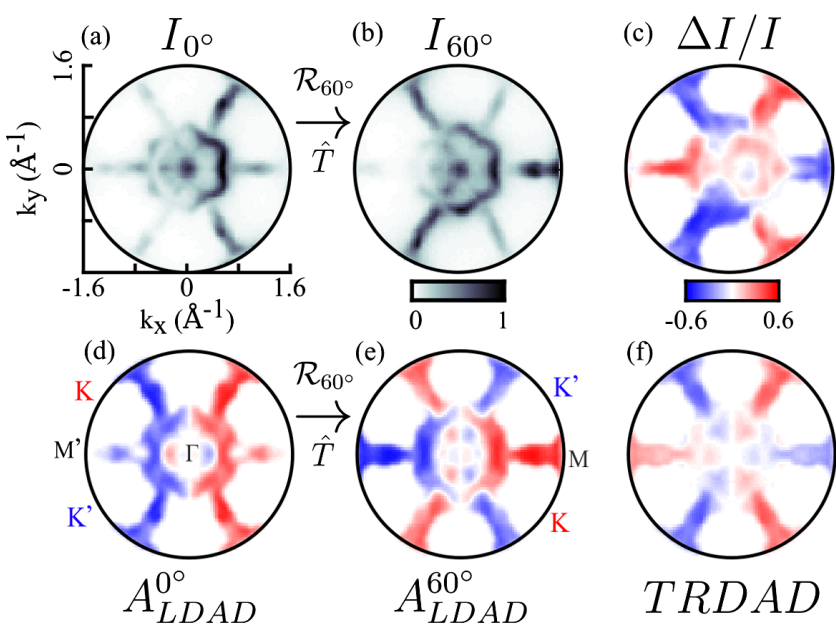

FIG. 2. Extraction of the time-reversal dichroism in photoelectron angular distributions (TRDAD). (a),(b) $I_{0^{\circ}}$ and $I_{60^{\circ}}$, the CECs for $E-E_{\mathrm{VBM}}=-1.60 \mathrm{eV}$, measured for two crystal orientations rotated by $60^{\circ}$ with respect to each other. (c) $\Delta I / I$, the raw normalized difference, i.e., $\left(I_{0^{\circ}}-I_{60^{\circ}}\right) /\left(I_{0^{\circ}}+\right.$ $\left.I_{60^{\circ}}\right)$ between CECs shown in (a) and (b). (d),(e) $A_{0^{\circ}}^{\mathrm{LDAD}}$ and $A_{\mathrm{LDAD}}^{60^{\circ}}$, the "left-right asymmetries," reflect the photoemission intensity asymmetry between the $k_{x}<0$ and $k_{x}>0$ hemispheres, for two crystal orientations, respectively, and are calculated using Eq. (1). (f) TRDAD [calculated using Eq. (2)] represents the component of $A_{\mathrm{LDAD}}^{0^{\circ} / 60^{\circ}}$ which is antisymmetric upon time reversal (i.e., upon $60^{\circ}$ azimuthal rotation of the crystal).

To investigate the microscopic origin of TRDAD, we perform state-of-the-art quantitative one-step photoemission calculations based on fully relativistic density functional theory. The one-step model of photoemission is implemented in the fully relativistic KKR method. The calculated photoemission signal is layer resolved and includes all matrix element effects such as experimental

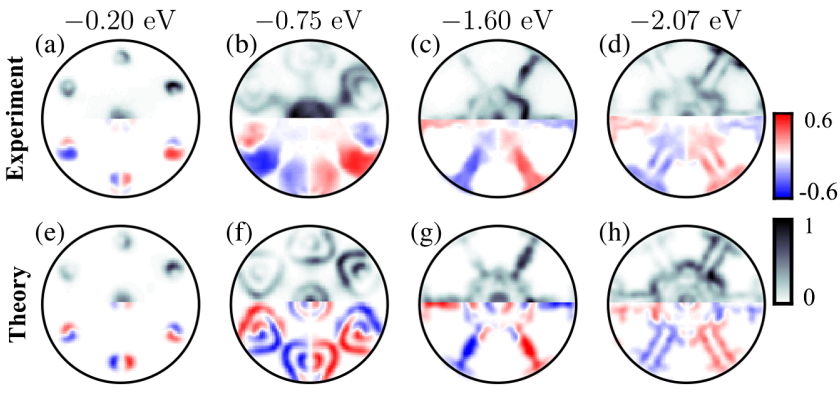

FIG. 3. Comparison between experimentally measured and theoretically (KKR) calculated TRDAD. In the upper hemisphere of each panel, the raw photoemission intensity, and in the lower hemisphere, TRDAD. (a)-(d) Experimentally measured photoemission intensity and TRDAD. (e)-(i) Calculated photoemission intensity and TRDAD. (a),(e) $E-E_{\mathrm{VBM}}=-0.20 \mathrm{eV}$, (b),(f) $E-E_{\mathrm{VBM}}=-0.75 \mathrm{eV}, \quad$ (c), (g) $E-E_{\mathrm{VBM}}=-1.60 \mathrm{eV}$, and (d),(i) $E-E_{\mathrm{VBM}}=-2.07 \mathrm{eV}$. The radius of each constant energy contour corresponds to $1.6 \AA^{-1}$. 


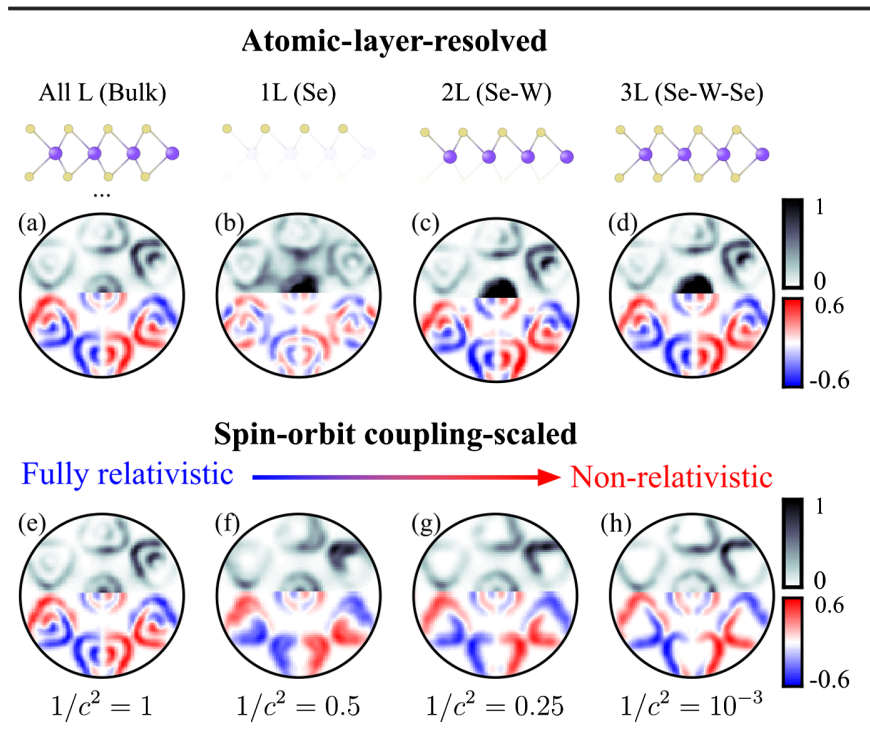

FIG. 4. Theoretical (KKR) investigations of the microscopic origin of the time-reversal dichroism. In the upper panels, we study the atomic-layer-resolved photocurrent and associated TRDAD. The signal is coming from (a) all layers (all L), (b) first Se atomic layer (Se), (c) first Se and W atomic layers (Se-W), and (d) first Se and $\mathrm{W}$ and second Se atomic layers (Se-W-Se). In the lower panels, we investigate the role of spin-orbit coupling on the photocurrent and associated TRDAD, by going from the fully relativistic to the nonrelativistic limit, upon modulating the speed of light in the calculations. (e) For standard speed of light (fully relativistic), which we defined as $1 / c^{2}=1$. (f)-(i) For enhanced speed of light, $1 / c^{2}=0.5,1 / c^{2}=0.25$, and $1 / c^{2}=10^{-3}$ (nonrelativistic limit), respectively. All the constant energy contours are taken at $E-E_{\mathrm{VBM}}=-0.75 \mathrm{eV}$, and their radii correspond to $1.6 \AA^{-1}$.

geometry, photon energy, polarization state, and final state effects (see Supplemental Material [27] and Refs. [21,22]).

The striking similarity between experimental and theoretical results (Fig. 3) confirms that the KKR method accurately describes the ground state properties of bulk $2 \mathrm{H}-$ $\mathrm{WSe}_{2}$ in an extended binding energy range and captures well subtleties of the photoemission process, including multiorbital interference effects. Now that the ability of the KKR method to quantitatively reproduce the experimentally measured signals is established, we want to strengthen our assertion that TRDAD in XUV photoemission probes hidden physical quantities, i.e., quantities that are nonvanishing in each constituent layer but that are vanishing in its inversion-symmetric counterpart (bulk). To do so, we investigated the atomic-layer-resolved photocurrent and associated TRDAD. In Fig. 4(a), the photoemission intensity and associated TRDAD emerging from all layers (bulk) are presented. The outer (VB1) and inner (VB2) bands around each $K / K^{\prime}$ valley show very similar dichroism, i.e., the same positive or negative (red and blue, respectively) TRDAD patterns. While the TRDAD signal coming from the topmost selenium (Se) atomic layer [Fig. 4(b)] is strongly different from the full calculation [Fig. 4(a)], including the photocurrent from the first tungsten (W) layer [Fig. 4(c)] is already enough to almost perfectly reproduce all features of the full calculation, which is in good agreement with the predicted $\mathrm{W} d$-type orbitals nature of the valence band at $K / K^{\prime}$ points. These calculations unambiguously confirm that TRDAD probes hidden physical quantities, which are modulated upon time reversal.

Next, we want to fully disentangle the signatures of spin and orbital textures in TRDAD. To do so, we have investigated the photoemission intensity and associated TRDAD in both the fully relativistic and nonrelativistic limit [vanishing spin-orbit coupling (SOC)]. Indeed, SOC is at the origin of the hidden spin polarization of the two topmost valence bands at $K / K^{\prime}$ and, thus, of the emergence of the peculiar spin texture in $2 \mathrm{H}$ TMDCs. In the limit where SOC vanishes (nonrelativistic limit), the two oppositely spin-polarized topmost valence bands at $K / K^{\prime}$ are merging together, leading to the annihilation of the spin polarization but to a conservation of the orbital texture. Because the dominant relativistic corrections scale with $1 / c^{2}$, where $c$ is the speed of light, a straightforward theoretical approach to go from the fully relativistic to the nonrelativistic limit, and thus to modify the strength of SOC, is to modulate the speed of light. In Fig. 4(e), one can see that, in the fully relativistic case, the topmost valence bands at $K / K^{\prime}$ are spin-orbit split and have similar TRDAD patterns, already suggesting its sensitivity to orbital texture. Strikingly, the nonrelativistic TRDAD from the degenerate band at $K / K^{\prime}$ is identical to the dichroism of the associated topmost valence band in the fully relativistic case. This observation is smoking-gun evidence that TRDAD is a powerful probe of the hidden orbital texture, which exists even in the absence of spin-orbit coupling and, thus, of spin texture.

Last, to get a more intuitive and comprehensive picture of the origin of TRDAD, we employ a third-nearest neighbor tight-binding (TB) model [15], which provides an excellent description of the electronic structure of $2 \mathrm{H}-$ $\mathrm{WSe}_{2}$ close to the $K / K^{\prime}$ points. As shown in Fig. 5(a), the TB model, for energies close to the valence band maximum, qualitatively reproduces the measured TRDAD. Our TB analysis unambiguously confirms that TRDAD originates from interference between $\left|d_{z^{2}}\right\rangle$ and $\left|d_{ \pm 2}\right\rangle$ orbitals, which is characterized by the orbital pseudospin texture (see Supplemental Material [27]). Moreover, as shown in Figs. 5(b) and 5(c), TRDAD is directly linked to the projection of the in-plane orbital pseudospin (black arrows) along the direction of the orbital vector field, which can be derived from the photoemission matrix elements (magenta arrows; see Supplemental Material [27] for details). Indeed, when the orbital pseudospin and the magenta arrow are parallel (antiparallel), TRDAD is positive (negative), while it vanishes when they are orthogonal. Our tight-binding analysis thus provides a direct relationship between the experimentally measured TRDAD and the orbital 

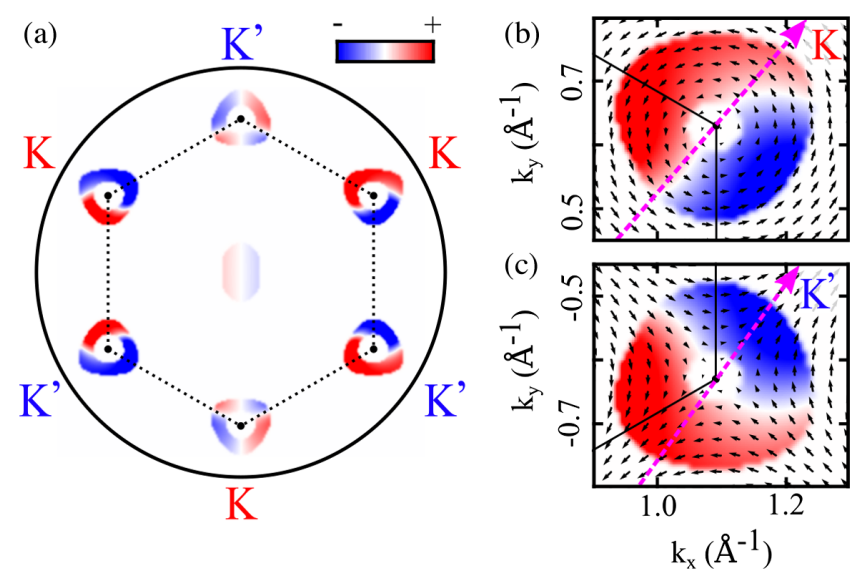

FIG. 5. Linking TRDAD and orbital pseudospin texture using a tight-binding framework. (a) TRDAD for an energy $E-$ $E_{\mathrm{VBM}}=-0.20 \mathrm{eV}$ computed using the tight-binding formalism described in Supplemental Material [27]. (b),(c) TRDAD and in-plane orbital pseudospin (black arrows) around the $K$ and $K^{\prime}$ valleys, respectively. The magenta dashed arrow represents the orientation of the orbital vector field, which can be derived from the photoemission matrix elements (more details can be found in Supplemental Material [27]). As shown in Eq. (7) of Supplemental Material [27], when the pseudospin texture is parallel (antiparallel) to the orbital vector field, we get a positive (negative) signal, while TRDAD vanishes if they are orthogonal. This relationship provides a direct link between the experimentally measured TRDAD and the in-plane orbital pseudospin.

pseudospin texture, a quantity of paramount importance in the emergence of Berry curvature and topological properties of matter.

In conclusion, we have introduced a novel and fully general robust observable in angle-resolved photoemission spectroscopy called time-reversal dichroism in photoelectron angular distributions, which probes the modulation of the photoemission intensity upon crystal rotation mimicking time reversal. We have demonstrated that the hidden orbital pseudospin texture of prototypical bulk $2 \mathrm{H}-\mathrm{WSe}_{2}$ leaves its imprint on TRDAD through the multiorbital interference process in photoemission. This robust observable is free of contributions from experimental geometry and is extremely stable against variation of the photon energy, contrary to most conventional dichroic ARPES signals, e.g., CDAD (see Supplemental Material [27]). Similar to the role of spin-resolved ARPES to experimentally elucidate complex momentum-space spin texture, we envision that TRDAD could emerge as the new standard observable to probe peculiar momentum-space orbital texture in complex multiorbital materials. Moreover, the extension of the approach to time-resolved TRDAD experiments is conceptually straightforward and will give access to the orbital texture of excited states and changes of topological properties of out-of-equilibrium states of matter, on ultrafast timescales [40-44].
All photoemission data used for the presented analysis are publicly available [45].

We thank Jürgen Henk and Karsten Horn for enlightening discussions at the early stage of the project. This work was funded by the Max Planck Society, the European Research Council (ERC) under the European Union's Horizon 2020 research and innovation program (Grant No. ERC-2015-CoG-682843), and the German Research Foundation (DFG) within the Emmy Noether program (Grant No. RE 3977/1) and through the SFB/TRR 227 "Ultrafast Spin Dynamics" (Projects No. A09 and No. B07). J. S. and J.M. thank the CEDAMNF project financed by the Ministry of Education, Youth and Sports of Czech Republic, Project No. CZ.02.1.01/0.0/0.0/15_003/ 0000358. J. B. and H. E. acknowledge financial support by the DFG via Projects No. Eb 158/32 and No. Eb 158/37. M. S. thanks the Alexander von Humboldt Foundation for its support with a Feodor Lynen scholarship. S. B. acknowledges financial support from the NSERC-Banting Postdoctoral Fellowships Program.

*beaulieu@fhi-berlin.mpg.de

jminar@ntc.zcu.cz

*ernstorfer@fhi-berlin.mpg.de

[1] K. F. Mak, K. He, J. Shan, and T. F. Heinz, Nat. Nanotechnol. 7, 494 (2012).

[2] H. Zeng, J. Dai, W. Yao, D. Xiao, and X. Cui, Nat. Nanotechnol. 7, 490 (2012).

[3] R. Bertoni, C. W. Nicholson, L. Waldecker, H. Hübener, C. Monney, U. De Giovannini, M. Puppin, M. Hoesch, E. Springate, R. T. Chapman, C. Cacho, M. Wolf, A. Rubio, and R. Ernstorfer, Phys. Rev. Lett. 117, 277201 (2016).

[4] M. Gmitra and J. Fabian, Phys. Rev. B 92, 155403 (2015).

[5] A. Avsar, D. Unuchek, J. Liu, O. L. Sanchez, K. Watanabe, T. Taniguchi, B. Özyilmaz, and A. Kis, ACS Nano 11, 11678 (2017).

[6] H. Zhang, C.-X. Liu, and S.-C. Zhang, Phys. Rev. Lett. 111, 066801 (2013).

[7] Z.-H. Zhu, C. N. Veenstra, G. Levy, A. Ubaldini, P. Syers, N. P. Butch, J. Paglione, M. W. Haverkort, I. S. Elfimov, and A. Damascelli, Phys. Rev. Lett. 110, 216401 (2013).

[8] P. D. C. King, S. McKeown Walker, A. Tamai, A. de la Torre, T. Eknapakul, P. Buaphet, S.-K. Mo, W. Meevasana, M. S. Bahramy, and F. Baumberger, Nat. Commun. 5, 3414 (2014).

[9] D. Go, D. Jo, C. Kim, and H.-W. Lee, Phys. Rev. Lett. 121, 086602 (2018).

[10] J.-H. Park, C. H. Kim, J.-W. Rhim, and J. H. Han, Phys. Rev. B 85, 195401 (2012).

[11] L. M. Canonico, T. P. Cysne, A. Molina-Sanchez, R. B. Muniz, and T. G. Rappoport, Phys. Rev. B 101, 161409(R) (2020).

[12] B. A. Bernevig, T. L. Hughes, and S.-C. Zhang, Phys. Rev. Lett. 95, 066601 (2005).

[13] V. T. Phong, Z. Addison, S. Ahn, H. Min, R. Agarwal, and E. J. Mele, Phys. Rev. Lett. 123, 236403 (2019). 
[14] S. Bhowal and S. Satpathy, Phys. Rev. B 101, 121112(R) (2020).

[15] G.-B. Liu, W.-Y. Shan, Y. Yao, W. Yao, and D. Xiao, Phys. Rev. B 88, 085433 (2013).

[16] The Pauli matrices act on the orbital space of $\left\{\left|d_{0}\right\rangle,\left|d_{ \pm 2}\right\rangle\right\}$ at $K / K^{\prime}$.

[17] X. Zhang, Q. Liu, J.-W. Luo, A. J. Freeman, and A. Zunger, Nat. Phys. 10, 387 (2014).

[18] J. M. Riley, F. Mazzola, M. Dendzik, M. Michiardi, T. Takayama, L. Bawden, C. Granerød, M. Leandersson, T. Balasubramanian, M. Hoesch, T. K. Kim, H. Takagi, W. Meevasana, P. Hofmann, M. S. Bahramy, J. W. Wells, and P. D. C. King, Nat. Phys. 10, 835 (2014).

[19] E. Razzoli, T. Jaouen, M.-L. Mottas, B. Hildebrand, G. Monney, A. Pisoni, S. Muff, M. Fanciulli, N. C. Plumb, V. A. Rogalev, V. N. Strocov, J. Mesot, M. Shi, J. H. Dil, H. Beck, and P. Aebi, Phys. Rev. Lett. 118, 086402 (2017).

[20] J. Tu, X. B. Chen, X. Z. Ruan, Y. F. Zhao, H. F. Xu, Z. D. Chen, X. Q. Zhang, X. W. Zhang, J. Wu, L. He, Y. Zhang, R. Zhang, and Y. B. Xu, Phys. Rev. B 101, 035102 (2020).

[21] H. Ebert, D. Ködderitzsch, and J. Minár, Rep. Prog. Phys. 74, 096501 (2011).

[22] J. Braun, J. Minár, and H. Ebert, Phys. Rep. 740, 1 (2018).

[23] M. Puppin, Y. Deng, C. W. Nicholson, J. Feldl, N. B. M. Schröter, H. Vita, P. S. Kirchmann, C. Monney, L. Rettig, M. Wolf, and R. Ernstorfer, Rev. Sci. Instrum. 90, 023104 (2019).

[24] K. Medjanik, O. Fedchenko, S. Chernov, D. Kutnyakhov, M. Ellguth, A. Oelsner, B. Schönhense, T. R. F. Peixoto, P. Lutz, C.-H. Min, F. Reinert, S. Däster, Y. Acremann, J. Viefhaus, W. Wurth, H. J. Elmers, and G. Schönhense, Nat. Mater. 16, 615 (2017).

[25] S. Beaulieu, S. Dong, N. Tancogne-Dejean, M. Dendzik, T. Pincelli, J. Maklar, R. P. Xian, M. A. Sentef, M. Wolf, A. Rubio, L. Rettig, and R. Ernstorfer, arXiv:2003.04059.

[26] J. Maklar, S. Dong, S. Beaulieu, T. Pincelli, M. Dendzik, Y. W. Windsor, R. P. Xian, M. Wolf, R. Ernstorfer, and L. Rettig, arXiv:2008.05829.

[27] See Supplemental Material at http://link.aps.org/ supplemental/10.1103/PhysRevLett.125.216404 for details about the ARPES experimental setup, the KKR calculations, and the derivation of the tight-binding model.

[28] H. Rostami, K. Volckaert, N. Lanata, S. K. Mahatha, C. E. Sanders, M. Bianchi, D. Lizzit, L. Bignardi, S. Lizzit, J. A. Miwa, A. V. Balatsky, P. Hofmann, and S. Ulstrup, Phys. Rev. B 100, 235423 (2019).

[29] Y. Liu, G. Bian, T. Miller, and T.-C. Chiang, Phys. Rev. Lett. 107, 166803 (2011).

[30] Y. H. Wang, D. Hsieh, D. Pilon, L. Fu, D. R. Gardner, Y. S. Lee, and N. Gedik, Phys. Rev. Lett. 107, 207602 (2011).
[31] S. Cho, J.-H. Park, J. Hong, J. Jung, B. S. Kim, G. Han, W. Kyung, Y. Kim, S.-K. Mo, J. D. Denlinger, J. H. Shim, J. H. Han, C. Kim, and S. R. Park, Phys. Rev. Lett. 121, 186401 (2018).

[32] M. Schüler, U. De Giovannini, H. Hübener, A. Rubio, M. A. Sentef, and P. Werner, Sci. Adv. 6, eaay2730 (2020).

[33] G. Schönhense, Phys. Scr. T T31, 255 (1990).

[34] N. A. Cherepkov and G. Schönhense, Europhys. Lett. 24, 79 (1993).

[35] A. Sterzi, G. Manzoni, A. Crepaldi, F. Cilento, M. Zacchigna, M. Leclerc, P. Bugnon, A. Magrez, H. Berger, L. Petaccia, and F. Parmigiani, J. Electron Spectrosc. Relat. Phenom. 225, 23 (2018).

[36] Y. Cao, J. A. Waugh, X.-W. Zhang, J.-W. Luo, Q. Wang, T. J. Reber, S. K. Mo, Z. Xu, A. Yang, J. Schneeloch, G. D. Gu, M. Brahlek, N. Bansal, S. Oh, A. Zunger, and D. S. Dessau, Nat. Phys. 9, 499 (2013).

[37] C.-H. Min, H. Bentmann, J. N. Neu, P. Eck, S. Moser, T. Figgemeier, M. Ünzelmann, K. Kissner, P. Lutz, R. J. Koch, C. Jozwiak, A. Bostwick, E. Rotenberg, R. Thomale, G. Sangiovanni, T. Siegrist, D. Di Sante, and F. Reinert, Phys. Rev. Lett. 122, 116402 (2019).

[38] S. Chernov, K. Medjanik, C. Tusche, D. Kutnyakhov, S. Nepijko, A. Oelsner, J. Braun, J. Minár, S. Borek, H. Ebert, H. Elmers, J. Kirschner, and G. Schönhense, Ultramicroscopy 159, 453 (2015).

[39] C. Tusche, P. Goslawski, D. Kutnyakhov, M. Ellguth, K. Medjanik, H. J. Elmers, S. Chernov, R. Wallauer, D. Engel, A. Jankowiak, and G. Schönhense, Appl. Phys. Lett. 108, 261602 (2016).

[40] M. A. Sentef, M. Claassen, A. F. Kemper, B. Moritz, T. Oka, J. K. Freericks, and T. P. Devereaux, Nat. Commun. 6, 7047 (2015).

[41] M. Claassen, C. Jia, B. Moritz, and T. P. Devereaux, Nat. Commun. 7, 13074 (2016).

[42] U. De Giovannini, H. Hübener, and A. Rubio, Nano Lett. 16, 7993 (2016).

[43] T. Oka and S. Kitamura, Annu. Rev. Condens. Matter Phys. 10, 387 (2019).

[44] M. Schüler, U. D. Giovannini, H. Hübener, A. Rubio, M. A. Sentef, T. P. Devereaux, and P. Werner, Phys. Rev. X 10, 041013 (2020).

[45] S. Beaulieu et al., Data for "Revealing Hidden Orbital Pseudospin Texture with Time-Reversal Dichroism in Photoelectron Angular Distributions", Zenodo, 10.5281/ zenodo.4023970 (2020).

Correction: The source information provided in Ref. [45] was set incorrectly during production and has been fixed. 